\title{
POLAR ACTIONS ON SYMMETRIC SPACES OF HIGHER RANK
}

\author{
ANDREAS KOLLROSS AND ALEXANDER LYTCHAK
}

\begin{abstract}
We show that polar actions of cohomogeneity two on simple compact Lie groups of higher rank, endowed with a biinvariant Riemannian metric, are hyperpolar. Combining this with a recent result of the second-named author, we are able to prove that polar actions induced by reductive algebraic subgroups in the isometry group of an irreducible Riemannian symmetric space of higher rank are hyperpolar. In particular, this result affirmatively settles the conjecture that polar actions on irreducible compact symmetric spaces of higher rank are hyperpolar.
\end{abstract}

\section{INTRODUCTION AND MAIN RESULTS}

A proper isometric action of a Lie group on a Riemannian manifold is called polar if there exists an immersed submanifold which meets every orbit and each such intersection is orthogonal. Such a submanifold is called a section of the Lie group action. If there is a section which is flat in its induced Riemannian metric, then the action is called hyperpolar.

A typical example of a polar action is the following. Consider the orthogonal group $\mathrm{O}(n)$, which acts on the space of real symmetric $n \times n$-matrices by conjugation. If this space is endowed with the scalar product given by $\langle X, Y\rangle=\operatorname{tr}(X Y)$, then the action is isometric. It is a well-known fact of linear algebra that every real symmetric matrix is conjugate to a diagonal matrix, which means that the linear subspace consisting of the diagonal matrices meets every orbit of the group action and it is easy to see that this subspace meets the orbits orthogonally. Hence the action is polar (in fact hyperpolar) with the subspace of diagonal matrices as a section. As this example suggests, one may think of the elements in a section as canonical representatives of the group orbits. The word "polar" is used because of the analogy to the usual polar coordinates on $\mathbb{R}^{2}$ which can be regarded as being

Date: November 1, 2018.

2000 Mathematics Subject Classification. 53C35, 57S15.

Key words and phrases. symmetric space, polar action, cohomogeneity two.

The second-named author was supported by a Heisenberg grant of the DFG and by the SFB "Groups, Geometry and Actions". 
given by the polar action of $\mathrm{O}(2)$ on $\mathbb{R}^{2}$, where the orbits are concentric circles around the origin and any straight line through the origin is a section. Indeed, polar actions on Riemannian manifolds are generalizations of the usual polar, cylindrical, or spherical coordinates in Euclidean space to general Riemannian manifolds. For surveys on polar actions, see [21] and [22].

Polar actions are closely related to Riemannian symmetric spaces. For example, if $G$ is the isometry group of a Riemannian symmetric space $M$ and

$$
K=\{g \in G \mid g \cdot p=p\}
$$

is the isotropy subgroup at a point $p \in M$ of the $G$-action on $M=G / K$, then the isotropy action of $M$, i.e. the action of $K$ on $M$, is polar, in fact hyperpolar.

Indeed, this action is well known from the theory of symmetric spaces [9]; the flats of a Riemannian symmetric space are the maximal totally geodesic and flat subspaces; all flats are congruent under the action of the isometry group; the rank of the symmetric space is defined to be the dimension of the flats. The sections of the isotropy action are given by those flats of $M$ which contain the point $p$. We say that a symmetric space is of higher rank if the rank of $M$ is greater than one.

The isotropy subgroup $K$ also acts on the tangent space $T_{p} M$ by the differentials of isometries. This linear representation of $K$ is called the isotropy representation of the symmetric space $M$; it is hyperpolar and the sections are the linear subspaces of $T_{p} M$ tangent to flats. Conversely, the main result of [5] says that all orthogonal representations of compact Lie groups on Euclidean space which are polar are given by this construction. More precisely, Dadok shows in his article [5] that any polar representation is orbit equivalent to an isotropy representation of a Riemannian symmetric space, i.e. after a suitable isometric identification of the two representation spaces, the connected components of the orbits coincide.

A special case of a Riemannian symmetric space is a connected compact Lie group $L$ equipped with a biinvariant Riemannian metric. These spaces are called symmetric spaces of Type II in [9]. In this case, left and right multiplication with arbitrary group elements are isometries and hence the group $L \times L$ acts isometrically on $L$ by

$$
(a, b) \cdot \ell:=a \ell b^{-1}
$$

In fact, the connected component of the isometry group of $L$ with this metric is covered by $L \times L=: G$. The isotropy subgroup $G_{e}$ at the identity element $e \in L$ is given by the diagonal $\{(\ell, \ell) \mid \ell \in L\}=: K$. The isotropy action of the symmetric space $L$ at the identity element $e$ is the restriction of (1.1) to $K$ and hence coincides with the action of $L$ on itself by conjugation. The sections of this hyperpolar action are the maximal tori of $L$. It is a well-known fact of Lie theory that if $T \subseteq L$ is 
a maximal torus, then any element of a connected compact Lie group is conjugate to some element in $T$, i.e. $T$ meets all the orbits of the action of $L$ on itself by conjugation (and it can be easily checked that $T$ meets the orbits orthogonally). Indeed, in a symmetric space of Type II, the flats containing the identity element $e$ are exactly the maximal tori. In particular, the rank of a symmetric space of Type II equals the dimension of the maximal tori.

Isotropy actions of Riemannian symmetric spaces provide examples of hyperpolar actions, but there is also a more general construction which involves two (in general) different symmetric spaces. Let $G$ be a compact semisimple Lie group and let $\sigma$ and $\tau$ be two involutive automorphisms. Let $H$ and $K$ be subgroups of $G$ such that $G_{o}^{\sigma} \subseteq H \subseteq G^{\sigma}$ and $G_{o}^{\tau} \subseteq K \subseteq G^{\tau}$, i.e. the groups $H$ and $K$ are open subsets of the fixed point sets of $\sigma$ and $\tau$. Assume that $G / K$ is endowed with the $G$-invariant metric induced by the negative of the Killing form of $\mathfrak{g}$. In this way, $G / K$ becomes a Riemannian symmetric space whose connected component of the isometry group is covered by $G$, see [9]. In particular, the closed subgroup $H \subseteq G$ naturally acts by isometries on $G / K$ and this action is in fact hyperpolar, see [8]. Actions which arise in this way are called Hermann actions since they were introduced in [10]. Of course, the isotropy action described above is just the special case of this construction where $H=K$

We show in this article that any polar action of cohomogeneity two on a simple compact classical Lie group $L$ of higher rank and endowed with a biinvariant Riemannian metric is hyperpolar. As we will point out below, this result provides the last step in the proof of the following theorem, which had been conjectured to be true by Biliotti in [2].

Theorem 1.1. Any polar action on an irreducible compact Riemannian symmetric space of higher rank which has an orbit of positive dimension is hyperpolar.

Note that we have to exclude the case of actions all of whose orbits are zerodimensional from the hypothesis of the theorem, since such actions are always polar (the whole space is the section) and they would otherwise provide technical counterexamples.

Theorem 1.1 has already been proved in a number of special cases. The first result in this connection was obtained by Brück [4], who showed that polar actions with a fixed point on irreducible Riemannian symmetric spaces (of compact or non-compact type) are hyperpolar. Podestà and Thorbergsson [20] have shown that polar actions on the complex quadric $\mathrm{SO}(n+2) / \mathrm{SO}(n) \mathrm{SO}(2)$ are hyperpolar. Their method relies on the fact that polar actions on Hermitian symmetric spaces are coisotropic, i.e. the normal spaces to principal orbits are totally real. (In fact, they have classified the coisotropic actions on the complex quadric.) Using the same method, this result 
has been generalized by Biliotti and Gori [3] to the complex Grassmannians and then by Biliotti [2] to all compact irreducible Hermitian symmetric spaces, leading Biliotti [2] to formulate the statement of Theorem 1.1 as a conjecture. However, this technique is not applicable to other (i.e. non-Hermitian) symmetric spaces and different methods are needed to prove the conjecture in a more general setting.

In the special cases of symmetric spaces of Type I, i.e. Riemannian symmetric spaces whose isometry group is a simple compact Lie group, the first-named author has obtained a complete classification of polar actions [12], proving Theorem [1.1 in this special case. The other class of irreducible compact Riemannian symmetric spaces are the symmetric spaces of Type II, i.e. compact Lie groups $L$ with biinvariant metric. These spaces have a non-simple isometry group; in fact, the connected component of the isometry group is covered by $L \times L$. The fact that the isometry group is non-simple makes it considerably more difficult to apply the methods of [12] in the case of symmetric spaces of Type II. However, the first-named author could still prove Theorem 1.1 in the special case of exceptional compact Lie groups with a biinvariant metric [13, again confirming the conjecture of Biliotti.

Recently, the second-named author obtained a result which generalizes Theorem 1.1 to the more general case of polar foliations, where the leaves of the foliation do not necessarily have to be homogeneous. He has shown that polar (singular Riemannian) foliations of irreducible compact symmetric spaces of higher rank are hyperpolar under the additional assumption that the codimension of the foliation is at least three [15]. (See [22] for a survey article on singular Riemannian foliations.) In order to finally complete the proof of Theorem 1.1, it now suffices to show that a polar action of cohomogeneity two on a classical compact Lie group $L$ endowed with a biinvariant Riemannian metric is hyperpolar if $\operatorname{rk}(L) \geq 2$. This is done in the present article by classifying all polar cohomogeneity two actions on classical compact Lie groups with biinvariant metric; all such actions turn out to be hyperpolar. See the last paragraph of Section 3 below for a summary of our proof.

Note that Theorem 1.1 does not generalize directly to non-compact symmetric spaces. In fact, there are counterexamples of polar actions with non-flat sections on non-compact symmetric spaces of higher rank, see [1, Proposition 4.2]. However, an analogous statement as in Theorem 1.1 still holds for actions on non-compact irreducible spaces if one requires the action to be given by a reductive algebraic subgroup of the isometry group. (For example, semisimple subgroups and compact subgroups of a semisimple Lie group are reductive algebraic subgroups, see [17].) Hence we also obtain the following.

Theorem 1.2. Let $M$ be an irreducible symmetric space. Let $H$ be a reductive algebraic subgroup of the isometry group of $M$. If the action of $H$ on $M$ is polar 
and has orbits of positive dimension, then the action is hyperpolar or $M$ is of rank one.

Theorem 1.2 follows immediately from Theorem 1.1 and [14, Theorem 5.1]. It was shown in [14] that hyperpolar actions of reductive algebraic subgroups in the isometry group of irreducible symmetric spaces are of cohomogeneity one or Hermann actions.

\section{Symmetric spaces of Type II AND their Isometry Groups}

Following [9, Ch. X], there are two types of irreducible compact Riemannian symmetric spaces. The spaces of Type I are those with simple compact isometry group; the spaces of Type II are given by simple compact Lie groups equipped with a biinvariant Riemannian metric. In the following, we study isometric group actions of compact Lie groups on Riemannian symmetric spaces of Type II.

Let $L$ be a compact connected simple Lie group equipped with a biinvariant Riemannian metric. Any such biinvariant metric is unique up to a constant scaling factor. To study polar actions, the choice of this scaling factors is of no relevance and we may thus assume $L$ is equipped with the biinvariant metric induced by the negative of the Killing form. Then $L$ together with this metric is a Riemannian symmetric space [9]; indeed, $L$ is a Riemannian homogeneous space (it acts isometrically on itself by left and right translations) and the inversion map $g \mapsto g^{-1}$ turns out to be an isometric geodesic symmetry at the identity element $e$ of $L$.

The connected component of the isometry group of this symmetric space is covered by $L \times L$, see [9]. To study (effective) polar actions on $L$, it therefore suffices to consider closed connected subgroups of $L \times L$ which act non-transitively. The following proposition shows that we may distinguish between two types of maximal non-transitive subgroups of $L \times L$.

Proposition 2.1. Let $H \subset L \times L$ be a connected closed subgroup acting nontransitively on $L$. Then $H$ is contained in one of the following subgroups of $L \times L$.

(i) A diagonal subgroup of the form

$$
\Delta^{\sigma} L:=\{(\ell, \sigma(\ell)) \mid \ell \in L\}
$$

where $\sigma$ is an automorphism of $L$.

(ii) A subgroup of the form

$$
H_{1} \times H_{2}:=\left\{\left(h_{1}, h_{2}\right) \mid h_{1} \in H_{1}, h_{2} \in H_{2}\right\},
$$

where $H_{1}$ and $H_{2}$ are closed connected proper subgroups of $L$.

Proof. Follows from [6, Theorem 15.1], see also [11, Section 2.1]. 


\section{CRITERION FOR POLARITY}

The following criterion for polarity of isometric actions on symmetric spaces of the compact type is well known, for a proof see for instance [12, Proposition 4.1]. By $H \cdot e K$ we denote the $H$-orbit through the point $e K \in G / K$ and by $N_{e K}(H \cdot e K)$ its normal space at the point $e K$.

Proposition 3.1. Let $G$ be a connected semisimple compact Lie group, let $\sigma$ be an involutive automorphism of $G$ and let $K \subset G$ be a closed subgroup such that $G_{o}^{\sigma} \subseteq K \subseteq G^{\sigma}$; in particular, $G / K$ is a Riemannian symmetric space of the compact type. Let $\mathfrak{g}=\mathfrak{k} \oplus \mathfrak{p}$ be the decomposition into eigenspaces of $d \sigma_{e}$ and identify $T_{e K} G / K$ with $\mathfrak{p}$ in the usual way. Let $H \subset G$ be a closed subgroup. Assume the element $e K$ of $G / K$ lies in a principal orbit of the $H$-action on $G / K$. Then the following are equivalent.

(i) The $H$-action on $G / K$ is polar with respect to any $G$-invariant Riemannian metric on $G / K$.

(ii) The subspace $\nu:=N_{e K}(H \cdot e K) \subseteq \mathfrak{p}$ is a Lie triple system such that the Lie algebra $\nu \oplus[\nu, \nu]$ generated by $\nu$ is orthogonal to $\mathfrak{h}$ with respect to the negative of the Killing form on $\mathfrak{g}$.

In particular, the $H$-action on $G / K$ is hyperpolar if and only if the subspace $\nu$ is an abelian subalgebra of $\mathfrak{p}$.

The proposition implies that the polarity of an action is determined on the Lie algebra level. The Lie triple system $\nu$ which appears in the proposition corresponds to the tangent space of a section in case of a polar action. (Note that sections of polar actions are always totally geodesic submanifolds [18.)

In this article, we will apply Proposition 3.1 exclusively to the special case of a Riemannian symmetric space of Type II. To this end, we use the homogeneous presentation $G / K$ of $L$ where we define $G:=L \times L$ and where the isotropy subgroup $K=G_{e}$ of the identity element $e \in L$ for the action (1.1) is the diagonal subgroup $\{(\ell, \ell) \mid \ell \in L\}$ of $L \times L$. In this case the spaces $\mathfrak{k}$ and $\mathfrak{p}$ as defined in Proposition 3.1 are of the following form

$$
\mathfrak{k}=\{(X, X) \mid X \in \mathfrak{l}\}
$$

and

$$
\mathfrak{p}=\{(X,-X) \mid X \in \mathfrak{l}\},
$$

where $\mathfrak{l}$ denotes the Lie algebra of $L$.

Henceforth we will assume that $L$ is a simple compact Lie group of rank greater than one and $H \subset L \times L$ is a closed connected subgroup which acts polarly with 
non-flat sections and with cohomogeneity two on $L$ by the restriction of (1.1) to $H$. We will prove that no such subgroup exists.

Our proof proceeds as follows. By Proposition 2.1 we may assume that the group $H$ is contained in a group of the form (2.1) or of the form (2.2). The first case has already been treated in [13] and hence may assume that $H$ is contained in a group of the form (2.2). In Lemma 5.1 we show that if $H$ is of the special form $H=\pi_{1}(H) \times \pi_{2}(H)$, where $\pi_{1}$ and $\pi_{2}$ denote the canonical projections on the first or second factor of $L \times L$, then the action is hyperpolar. Thus we may assume in what follows that $H$ is a proper subgroup of $\pi_{1}(H) \times \pi_{2}(H)$. Moreover, since the action of $\pi_{1}(H) \times \pi_{2}(H)$ is not orbit equivalent to the $H$-action, it is either of cohomogeneity one or transitive. However, in Section 6 we show that subactions of cohomogeneity one actions cannot be polar, ruling out the first alternative. It remains to consider the case where $\pi_{1}(H) \times \pi_{2}(H)$ acts transitively on $L$ by (1.1) and where both $\pi_{1}(H)$ and $\pi_{2}(H)$ are proper subgroups of $L$. Using the classification of transitive actions [16], it is shown in Section 7 that there is no such action, completing the proof of Theorem 1.1.

\section{Subactions of $\sigma$-ACtions}

We start our classification with actions by (closed subgroups of) diagonal subgroups in $L$.

Lemma 4.1. Let $L$ be a simple compact Lie group and let $\sigma$ be an automorphism of $L$. If a closed connected subgroup $H$ of $\Delta^{\sigma} L$ acts polarly on the simple compact connected Lie group $L$ of rank greater than one, then $H=\Delta^{\sigma} L$ or $H=\{e\}$; in particular, the action of $H$ on $L$ is hyperpolar or trivial.

Proof. [13, Proposition 12].

Note that the action of $\Delta^{\sigma} L$ on $L$ is well known to be hyperpolar. These actions were named $\sigma$-actions in [8]. From now on, it suffices to consider those subgroups of $L \times L$ which are contained in groups of the form (2.2). We will first look at a special case.

\section{Groups Without diagonal Factors}

In this section we consider the special case where the subgroup $H \subset L \times L$ is such that $H=\pi_{1}(H) \times \pi_{2}(H)$. It is more or less an immediate consequence of Proposition 3.1 that such an action is hyperpolar. 
Lemma 5.1. Let $L$ be a simple compact Lie group and let $H_{1}, H_{2} \subset L$ be closed subgroups. Assume that the subgroup

$$
H_{1} \times H_{2}=\left\{\left(h_{1}, h_{2}\right) \mid h_{1} \in H_{1}, h_{2} \in H_{2}\right\} \subseteq L \times L
$$

acts polarly and with cohomogeneity two on $L$ by the restriction of the action (1.1). Then the $\mathrm{H}_{1} \times \mathrm{H}_{2}$-action on $\mathrm{L}$ is hyperpolar.

Proof. We may assume that the identity element $e \in L$ lies in a principal orbit by replacing $H$ with a suitable conjugate group, if necessary. Let $\nu$ be the normal space at the point $e$ to the $H$-orbit through $e$ as defined in Proposition 3.1 (ii). By the hypothesis, the $H$-action is of cohomogeneity two, hence there are two linearly independent vectors $v, w \in \nu$. Furthermore, since the $H$-action is polar, it follows from Proposition 3.1 that $[v, w]$ is orthogonal to $\mathfrak{h}$. Since the elements of $\mathfrak{p}$ (and hence of $\nu$ ) are of the special form (3.2), we have $v=(X,-X), w=(Y,-Y)$ for some vectors $X, Y \in \mathfrak{l}$. Consequently, we have $[v, w]=[(X,-X),(Y,-Y)]=$ $([X, Y],[X, Y])$. By our hypothesis, the group $H$ is of the special form (5.1) and hence an element $(A, B) \in \mathfrak{l} \times \mathfrak{l}$ is orthogonal to $\mathfrak{h}$ if and only if $A$ is orthogonal to $\mathfrak{h}_{1}$ and $B$ is orthogonal to $\mathfrak{h}_{2}$. In particular, since the vector $[v, w]=([X, Y],[X, Y])$ is orthogonal to $\mathfrak{h}$, we also have that the vector $([X, Y],-[X, Y]) \in \mathfrak{p}$ is orthogonal to $\mathfrak{h}$ and hence it is contained in the normal space $\nu$.

Assume now the three vectors $X, Y$ and $[X, Y]$ in $\mathfrak{l}$ are linearly independent. Then it follows that the three vectors $(X,-X),(Y,-Y),([X, Y],-[X, Y])$ in $\nu$ are linearly independent, too. Since $\nu$ is the normal space at a principal orbit, it follows that the cohomogeneity of the $H$-action is at least three, contradicting our hypothesis.

Thus it follows that the bracket $[X, Y]$ is contained in the span of $X$ and $Y$. Hence the vectors $X$ and $Y$ span a two-dimensional subalgebra of $\mathfrak{l}$. However, the Lie group $L$ is compact and hence any subalgebra of $\mathfrak{l}$ is reductive. In particular, any subalgebra of $\mathfrak{l}$ decomposes as a direct sum of an abelian and a semisimple Lie algebra. By the classification of semisimple real Lie algebras, it is known that any simple Lie algebra (and hence any non-trivial semisimple Lie algebra) is at least of dimension three. It follows that the Lie algebra spanned by $X$ and $Y$ is abelian and Proposition 3.1 implies that the action is hyperpolar.

As a consequence of Lemmas 4.1 and 5.1 we obtain the following.

Lemma 5.2. Let $L$ be a simple compact Lie group and assume $H \subset L \times L$ is a closed connected subgroup whose action on $L$ is of cohomogeneity two and polar with a non-flat section. Then there are closed connected proper subgroups $\mathrm{H}_{1}$ and $\mathrm{H}_{2}$ of $L$ such that $H$ is contained in $H_{1} \times H_{2}$ and such that the action of $H_{1} \times H_{2}$ on $L$ given by $\left(h_{1}, h_{2}\right) \cdot \ell:=h_{1} \ell h_{2}^{-1}$ is of cohomogeneity one or transitive. 
Proof. Define $H_{1}$ and $H_{2}$ to be the images of $H$ under the natural projections onto the first and second factors of $L \times L$. Then $H_{1}$ and $H_{2}$ are compact connected subgroups of $L$ and $H$ is contained in $H_{1} \times H_{2}$. If $H_{1}=L$ or $H_{2}=L$, then $\mathfrak{h}$ contains an ideal isomorphic to $\mathfrak{l}$ and it follows from Lemma 2.1 that $H$ is contained in a subgroup of the form (2.1). Hence it follows from Lemma 4.1 that the $H$ action on $L$ is hyperpolar, a contradiction. Thus $H_{1}$ and $H_{2}$ are proper subgroups of $L$. Since we have $H \subseteq H_{1} \times H_{2}$, it follows that the $H_{1} \times H_{2}$-action on $L$ is cohomogeneity less or equal to two. If the cohomogeneity is two, then the $H$-action and the $H_{1} \times H_{2}$-action on $L$ are orbit equivalent, leading to a contradiction, since then by Lemma 5.1 the $H_{1} \times H_{2}$-action is hyperpolar. Thus the $H_{1} \times H_{2}$-action on $L$ is of cohomogeneity one or transitive.

From now on, we may assume that the $H$-action on $L$ is a subaction of a transitive or cohomogeneity one action given by a subgroup of the form (2.2). (By the term subaction we refer to an action which is given by restricting a Lie group action to a closed subgroup.) We will prove in the next two sections that no such subaction exists.

\section{Subactions of cohomogeneity one aCtions}

Proposition 6.1. Let $M$ be a compact irreducible symmetric space of higher rank with a polar and not hyperpolar action of a group $H$ of cohomogeneity two. Then $H$ is not a subgroup of a larger group $H^{\prime}$ which acts on $M$ isometrically and with cohomogeneity one.

Proof. Assume that such a group $H^{\prime}$ does exist. We may assume that $H$ and $H^{\prime}$ are connected. Denote by $\Delta$ the quotient space $M / H$. By polarity of the action of $H$, $\Delta$ is a finite quotient of a section $\Sigma$, which is a totally geodesic submanifold of $M$ and hence a two-dimensional symmetric space. By assumption $\Sigma$ is non-flat. Thus it is either the round sphere or the round projective space, which, after rescaling, may be assumed to be of constant curvature one. In any case, $\Delta$ is the quotient of the universal covering $S^{2}$ of $\Sigma$ by a finite Coxeter group $W$.

In [15, Section 7] it is shown that if the Coxeter group $W$ is reducible, the space $M$ must be of rank one. Thus we may assume that $W$ is irreducible. Then $\Delta$ is a spherical triangle with angles $\pi / 2, \pi / 3, \pi / m$, with $m=3$ or $m=4$. (In fact, the case $m=3$ can be excluded using the ideas of [15], cf. [7], but this will not be used in the sequel). Hence, the triangle $\Delta$ has two vertices $x$ and $y$ such that the angles at these points are equal to $\pi / m$ with (possibly different) $m>2$.

Let $p \in M$ be any point in the $H$-orbit corresponding to $x$. Then the isotropy group $H_{p}$ acts on the normal space $V$ to the orbit $H \cdot p$ with cohomogeneity two, 
such that the quotient $V / H_{p}$ is the tangent space to $\Delta$ at $x$, i.e. the cone over the interval of length $\pi / m$. Hence the action of $H_{p}$ on $V$ is irreducible. Therefore, the larger isotropy group $H_{p}^{\prime}$ of $H^{\prime}$ at $p$ cannot act on a proper non-trivial subspace of $V$. It follows that the normal space to the orbit $H^{\prime} \cdot p$ at $p$ coincides with $V$. Thus the orbits $H^{\prime} \cdot p$ and $H \cdot p$ coincide.

The same argument works for a point $q$ over the vertex $y$. Thus we deduce that the orbits of $H$ and of $H^{\prime}$ through $p$ and $q$ coincide. Therefore, $H \cdot p$ and $H \cdot q$ are the only singular orbits of the cohomogeneity-one-action of $H^{\prime}$ on $M$.

Take any regular point $o$ in the section $\Sigma$ from above. Then this point $o$ must lie on a shortest $H^{\prime}$-horizontal geodesic from $H \cdot p$ to $H \cdot q$. This geodesic is also $H$-horizontal, hence contained in $\Sigma$. For a generic choice of $o$ in $\Sigma$, we obtain a contradiction.

Remark 6.2. If, under the assumptions of Proposition 6.1, the space $M$ is reducible and $H^{\prime}$ acts with cohomogeneity one, then (possibly after enlarging the group $H$ without changing the orbit equivalence class) we are in the following situation. The space $M$ splits as $M=M_{1} \times M_{2}$ and $H$ splits as $H_{1} \times H_{2}$, such that $H_{i}$ acts trivially on $M_{2-i}$. Moreover, $H_{2}$ acts transitively on $M_{2}$ and $M_{1}$ is of rank one, see [15, Section 7].

\section{Subactions of transitive aCtions}

Lemma 7.1. Let $L$ be a simple compact Lie group of rank greater than one. Then there is no closed subgroup $H \subset L \times L$ such that the $H$-action on $L$ is polar of cohomogeneity two with a non-flat section and such that the action of $\pi_{1}(H) \times \pi_{2}(H)$ on $L$ is transitive.

Proof. Consider the ideals $\mathfrak{h}_{1}^{\prime}:=\operatorname{ker}\left(\pi_{2} \mid \mathfrak{h}\right)$ and $\mathfrak{h}_{2}^{\prime}:=\operatorname{ker}\left(\pi_{1} \mid \mathfrak{h}\right)$ of $\mathfrak{h}$, where $\pi_{1}$ and $\pi_{2}$ are the natural projections onto the two simple factors of the Lie algebra of $L \times L$. Let $\mathfrak{h}_{\Delta}$ be an ideal which is complementary to $\mathfrak{h}_{1}^{\prime}+\mathfrak{h}_{2}^{\prime}$ in $\mathfrak{h}$. Then we have $\pi_{1}(\mathfrak{h})=$ $\pi_{1}\left(\mathfrak{h}_{\Delta}\right) \oplus \mathfrak{h}_{1}^{\prime}$ and $\pi_{2}(\mathfrak{h})=\pi_{2}\left(\mathfrak{h}_{\Delta}\right) \oplus \mathfrak{h}_{2}^{\prime}$. Since $\mathfrak{h}$ is a proper subalgebra of $\pi_{1}(\mathfrak{h}) \times \pi_{2}(\mathfrak{h})$, we have $\mathfrak{h}_{\Delta} \neq 0$.

All transitive actions of groups $H_{1} \times H_{2}$ on simple compact Lie groups $L$ given by (1.1), where $H_{1}, H_{2} \subset L$ are closed proper subgroups, were determined by Oniščik [16]; the result is given in Table 1. Inspection of the table shows that for $L$ simple there is only one case where the Lie algebras of $H_{1}$ and $H_{2}$ have a nontrivial isomorphic ideal, namely the case of the $\operatorname{Spin}(7) \times \mathrm{SO}(7)$-action on $\mathrm{SO}(8)$. However, in this case $\mathfrak{h}=\mathfrak{h}_{\Delta}$ would be isomorphic to the 21-dimensional Lie algebra $\mathfrak{s o}(7)$, obviously contradicting the assumption that $H$ acts on the 28-dimensional Lie group $\mathrm{SO}(8)$ with cohomogeneity two. 


\begin{tabular}{|c|c|c|}
\hline$H_{1}$ & $L$ & $\mathrm{H}_{2}$ \\
\hline$\overline{\mathrm{Sp}(n)}$ & $\overline{\mathrm{SU}(2 n)}$ & $\begin{array}{c}\mathrm{S}(\mathrm{U}(2 n-1) \mathrm{U}(1)) \\
\mathrm{SU}(2 n-1)\end{array}$ \\
\hline$\overline{\mathrm{SO}(2 n-1)}$ & $\mathrm{SO}(2 n)$ & $\begin{array}{c}\mathrm{U}(n) \\
\mathrm{SU}(n)\end{array}$ \\
\hline $\mathrm{SO}(4 n-1)$ & $\mathrm{SO}(4 n)$ & $\begin{array}{c}\operatorname{Sp}(n) \cdot \operatorname{Sp}(1) \\
\operatorname{Sp}(n) \cdot U(1) \\
\operatorname{Sp}(n)\end{array}$ \\
\hline $\mathrm{G}_{2}$ & $\mathrm{SO}(7)$ & $\mathrm{SO}(6)$ \\
\hline $\mathrm{G}_{2}$ & $\overline{\mathrm{SO}(7)}$ & $\begin{array}{c}\mathrm{SO}(5) \mathrm{SO}(2) \\
\mathrm{SO}(5)\end{array}$ \\
\hline $\operatorname{Spin}(7)$ & $\mathrm{SO}(8)$ & $\mathrm{SO}(7)$ \\
\hline $\operatorname{Spin}(9)$ & $\mathrm{SO}(16)$ & $\mathrm{SO}(15)$ \\
\hline
\end{tabular}

TABLE 1. Transitive actions.

Remark 7.2. It should be noted that Table 1 has to be interpreted on the Lie algebra level. Indeed, e.g. the transitive actions on $\mathrm{SO}(8)$ of $\mathrm{Spin}(7) \times[\mathrm{SO}(6) \mathrm{SO}(2)]$ and of $\mathrm{Spin}(7) \times[\mathrm{SO}(5) \mathrm{SO}(3)]$ correspond to the actions of $\mathrm{SO}(7) \times \mathrm{U}(4)$ and $\mathrm{SO}(7) \times$ $[\operatorname{Sp}(2) \cdot \operatorname{Sp}(1)]$ under a triality automorphism of $\operatorname{Spin}(8)$, cf. [11, Proposition 3.3]. In particular, some entries of [16, Table 7] do not appear in our Table 1] exactly for this reason.

\section{Concluding Remarks}

It follows from the main result of [15] that polar actions on compact irreducible Riemannian symmetric spaces of higher rank are hyperpolar if the cohomogeneity is greater than two. In this article, we have shown that polar actions of cohomogeneity two on the classical compact Lie groups of higher rank with biinvariant Riemannian metrics are hyperpolar. Without restriction on the cohomogeneity, this was shown in [12] for symmetric spaces of Type I and for exceptional symmetric spaces of Type II in [13. Thus we have now completed the proof of Theorem 1.1. In particular, this finally settles the conjecture of Biliotti [2] affirmatively.

It now follows from the results of [11] that any polar action of a compact Lie group on a compact irreducible Riemannian symmetric space of higher rank is of cohomogeneity one or orbit equivalent to a Hermann action (it can be both, as there are many Hermann actions of cohomogeneity one). See [11, Theorem B] for the classification of cohomogeneity one actions up to orbit equivalence. Let us recall the two types of Hermann actions in the case where the symmetric space $G / K$ is 
isometric to a simple compact Lie group $L$ with biinvariant Riemannian metric. In this case we may assume $G=L \times L$ and any involutive automorphism of $G$ is either of the form $\left(\ell_{1}, \ell_{2}\right) \mapsto\left(\sigma^{-1}\left(\ell_{2}\right), \sigma\left(\ell_{1}\right)\right)$, where $\sigma$ is an automorphism of $L$ (of arbitrary order) or of the form $\left(\ell_{1}, \ell_{2}\right) \mapsto\left(\sigma\left(\ell_{1}\right), \tau\left(\ell_{2}\right)\right)$ where both $\sigma$ and $\tau$ are involutive automorphisms of $L$. In the first case, the fixed point set of the involution is $\Delta^{\sigma}=\{(\ell, \sigma(\ell)) \mid \ell \in L\} \subset G$. In the latter case, the fixed point set is given by the cartesian product of the fixed point sets $L^{\sigma} \times L^{\tau}$.

As stated in Lemma 4.1, any subgroup of $L \times L$ whose action on $L$ is orbit equivalent to the action of $\Delta^{\sigma}$ is actually conjugate to $\Delta^{\sigma}$. However, in case of the action of $L^{\sigma} \times L^{\tau}$ on $L$, there are many examples of orbit equivalent subgroups, see [12].

On the compact rank-one symmetric spaces, polar actions have been classified in [19].

\section{REFERENCES}

[1] J. Berndt, J.C. Díaz-Ramos and H. Tamaru, Hyperpolar homogeneous foliations on symmetric spaces of noncompact type, J. Differential Geom. 86, (2), 191-236 (2010)

[2] L. Biliotti, Coisotropic and polar actions on compact irreducible Hermitian symmetric spaces, Trans. Amer. Math. Soc. 358, 3003-3022 (2006)

[3] L. Biliotti and A. Gori, Coisotropic actions on complex Grassmannians, Trans. Amer. Math. Soc. 357, 1731-1751 (2005)

[4] M. Brück, Äquifokale Familien in symmetrischen Räumen. (Equifocal families in symmetric spaces) (German), Doctoral Dissertation, Universität zu Köln (1998)

[5] J. Dadok, Polar coordinates induced by actions of compact Lie groups, Trans. Amer. Math. Soc. 288, 125-137 (1985)

[6] E.B. Dynkin, Semisimple subalgebras of the semisimple Lie algebras, (Russian). Mat. Sbornik 30, 349-462 (1952); English translation: Amer. Math. Soc. Transl. Ser. 2, 6, 111244 (1957)

[7] F. Fang, K. Grove and G. Thorbergsson, Tits geometry and positive curvature, arXiv:1205.6222v1 [math.DG] (2012)

[8] E. Heintze, R. Palais, C.L. Terng and G. Thorbergsson, Hyperpolar actions on symmetric spaces, Geometry, topology and physics for Raoul Bott, ed. S.-T. Yau, International Press, Boston, Mass. (1994)

[9] S. Helgason, Differential geometry, Lie groups and symmetric spaces. Academic Press, New York (1978)

[10] R. Hermann, Variational completeness for compact symmetric spaces, Proc. Amer. Math. Soc. 11, 544-546 (1960)

[11] A. Kollross, A classification of hyperpolar and cohomogeneity one actions, Trans. Amer. Math. Soc. 354, 571-612 (2002)

[12] A. Kollross, Polar actions on symmetric spaces, J. Differential Geom. 77 (3), 425-482 (2007)

[13] A. Kollross, Low cohomogeneity and polar actions on exceptional compact Lie groups, Transformation Groups, 14, No.2, 387-415 (2009) 
[14] A. Kollross, Duality of symmetric spaces and polar actions, Journal of Lie Theory, 21, 961-986 (2011)

[15] A. Lytchak, Polar foliations on symmetric spaces, arXiv:1204.2923v1 [math.DG] (2012)

[16] A.L. Oniščik, Inclusion relations among transitive compact transformation groups, (Russian). Trudy Mosk. Mat. Obshch. 11, 199-242, (1962); English translation: Amer. Math. Soc. Transl. Ser. 2, 50, 5-58 (1966)

[17] A.L. Oniščik and E.B. Vinberg, (Eds.), Lie groups and Lie algebras III, Encyclopaedia of Mathematical Sciences 41, Springer, Berlin (1994).

[18] R. Palais and C.-L. Terng, A general theory of canonical forms, Trans. Amer. Math. Soc. 300, 771-789 (1987)

[19] F. Podestà and G. Thorbergsson, Polar actions on rank-one symmetric spaces, J. Differential Geom. 53 (1), 131-175 (1999)

[20] F. Podestà and G. Thorbergsson, Polar and coisotropic actions on Kähler manifolds, Trans. Amer. Math. Soc. 354, 1749-1757 (2002)

[21] G. Thorbergsson, Transformation groups and submanifold geometry, Rend. Mat., Ser. VII, 25, 1-16 (2005)

[22] G. Thorbergsson, Singular Riemannian foliations and isoparametric submanifolds, Milan J. Math. 77, 127-150 (2010)

Universität Stuttgart, Fachbereich Mathematik, Institut für Geometrie und Topologie, Pfaffenwaldring 57, D-70569 Stuttgart, Germany

Universität Zu Köln, Mathematisches Institut, Weyertal 86-90, D-50931 Köln, GERMANY

E-mail address: kollross@mathematik.uni-stuttgart.de

E-mail address: alytchak@math. uni-koeln.de 